\title{
Diseño de sistemas de medición de desempeño de proveedores: experiencias de un caso de estudio
}

\author{
Nicole Suclla Fernandez,*, Luiz Felipe Scavarda ${ }^{\mathrm{b}}$, \\ Adriana Leiras ${ }^{c}$, Silvio Hamacher ${ }^{\mathrm{d}}$ \\ a,*nicolesf@aluno.puc-rio.br, PUC-Rio, Brasil \\ blf.scavarda@puc-rio.br, PUC-Rio, Brasil \\ cadrianaleiras@yahoo.com.br, PUC-Rio, Brasil \\ dhamacher@puc-rio.br, PUC-Rio, Brasil
}

\begin{abstract}
Resumen
La evaluación de los proveedores es importante para las organizaciones debido al papel preponderante que éstos cumplen en la dinámica de las cadenas de suministros y a la importancia estratégica que las funciones de compra tienen a raíz de la tercerización de los procesos que no forman parte del know-how de la empresa. No obstante, la literatura académica carece de trabajos concernientes al diseño e implementación de sistemas de medición de desempeño de proveedores (SMDP). En ese contexto, este artículo presenta los factores más importantes del diseño de un SMDP, resaltando, a través de un caso de estudio, los cuidados y recomendaciones que deben ser considerados. Además del detalle del método y los resultados de su aplicación, el artículo presenta el análisis de los procesos y atributos que definen el diseño del SMDP propuesto.
\end{abstract}

Palabras-clave

Sistemas de medición de desempeño. Cadenas de suministros. Proveedores. Evaluación del desempeño de proveedores.

\section{Introducción}

Los indicadores de desempeño son fundamentales en el ciclo de planeamiento y control estratégico de la empresa, siendo objeto del creciente interés entre profesionales e investigadores académicos (JUSOH; IBRAHIM; ZAINUDDIN, 2008). Estos proporcionan un medio para generar la información necesaria para tomar decisiones, permitiendo que la alta gerencia sepa, en función del desempeño real del negocio, cuando intervenir (NEELY; GREGORY; PLATTS, 1995; NEELY et al., 1997). Un conjunto de indicadores de desempeño puede ser considerado un sistema de medición cuando en su amplitud es incorporado el estudio del software o plataforma de implementación, la estructuración de bancos de datos y la definición de procedimientos organizacionales que permitan medir el desempeño en forma completa y consistente (NEELY; GREGORY; PLATTS, 1995; NEELY et al., 2002; LOHMAN; FORTUIN; WOUTERS, 2004). Los sistemas de medición de desempeño deben ser capaces de generar datos que permitan el análisis del desempeño pasado y el planeamiento del desempeño futuro, evidenciando la relación existente entre las medidas resultantes y las decisiones tomadas (NEELY et al., 1996).

La ausencia de criterios bien definidos, que realmente describan el desempeño de individuos y de la propia organización, evitan que los esfuerzos gerenciales sean fructíferos para la motivación de sus funcionarios y para alcanzar los objetivos trazados (GLOBERSON, 1985). La definición de tales criterios es un desafío que aumenta con la complejidad y diversidad de las tareas a evaluar, motivo por el cual, implementar un sistema de medición de desempeño adecuado para una cadena de suministros es un reto gerencial (LAMBERT; POHLEN, 2001; HARRISON; LEE; NEALE, 2003; SHEPHERD; GÜNTER, 2006). Cada eslabón de la cadena de suministros (supply chain) es caracterizado por la interacción entre proveedores y compradores, donde, desde el punto de vista gerencial de la 
empresa compradora, existe una exigencia continua para trabajar con proveedores de alto desempeño. La importancia de trabajar conjuntamente con los proveedores y de utilizar métodos adecuados para su evaluación aumenta proporcionalmente con la tendencia de tercerizar actividades que no forman parte del know-how productivo de la organización (COLLINS; BECHLER; PIRES, 1997; TATE; van DER VALK, 2008). En ese contexto, las actividades del departamento de compras toman características estratégicas en la elección, control y manutención de los varios tipos de proveedores contratados por la empresa (SPEKMAN; KAMAUFF; SALMOND, 1994; WAGNER; JOHNSON, 2004; KOCABASOGLU; SURESH, 2006; WISNER; STANLEY, 2007).

El desafío en la evaluación de proveedores consiste en diseñar un sistema de medición de desempeño que incluya un conjunto de indicadores SPE (Supplier Performance Evaluation) capaces de permitir que los gerentes identifiquen los problemas en los procesos de la empresa que son directa e indirectamente afectados por el desempeño de sus proveedores (BEAMON, 1998; HOEK, 1998; TRACEY; TAN, 2001; CEBI; BAYRAKTAR, 2003). Todavía existe insuficiente literatura académica que aborde las dificultades comúnmente encontradas en la implementación de sistemas de medición de desempeño de proveedores (SMDP) (HARRISON; LEE; NEALE, 2003). De esta manera, la necesidad de trabajos que documenten y ejemplifiquen los métodos utilizados en el diseño de SMDP aplicados a diferentes realidades organizacionales, define un interesante campo de estudio a explorar.

En este escenario, el presente artículo tiene como objetivo exhibir los factores más importantes del diseño de un SMDP en una empresa multinacional de grande porte, fuerte presencia en mercados latinos y que no contaba con una evaluación formal del desempeño de sus proveedores. El artículo resalta por medio de un caso de estudio los cuidados y recomendaciones que deben ser considerados en la conjunción de los conceptos teóricos de medición de desempeño y las diferentes necesidades estratégicas y operacionales de la empresa en análisis. Por motivos de confidencialidad ésta empresa será llamada de CLAVE.

El artículo está organizado en cinco secciones, siendo que la primera sección introductoria es la recientemente presentada. La sección 2 expone los fundamentos teóricos relevantes para conducir la investigación. La sección 3 presenta el método de investigación utilizado. La sección 4 expone el diseño y análisis del SMDP en un caso de estudio. La sección 5 presenta la conclusión del artículo.

\section{Sistemas de medición de desempeño}

Los indicadores de desempeño forman la métrica utilizada para cuantificar la eficiencia y/o eficacia de una acción en relación a una meta previamente definida (FORTUIN, 1988; NEELY et al., 1996). Los indicadores incluidos en un sistema de medición de desempeño (SMD) deben ser coherentes con los objetivos y actividades de todos los niveles organizacionales: estratégico o gerencial, táctico y operacional (DO LAGO ATTADIA; MARTINS, 2003; GUNASEKARAN; PATEL; McGAUGHEY, 2004). En ese sentido, un SMD procura que los gestores tomen decisiones basándose en los resultados de la colecta de datos, compilación, análisis y diseminación de informaciones apropiadas para la organización (NEELY et al., 1997; NEELY et al., 2002; LOHMAN; FORTUIN; WOUTERS, 2004). Tales datos o medidas, alimentan los indicadores del SMD y pueden ser de carácter cuantitativo y/o cualitativo. Las mediciones cuantitativas son basadas en cálculos numéricos obtenidos a partir de estadísticas del proceso productivo. Las mediciones cualitativas dependen de la apreciación del evaluador, teniendo un alto grado de subjetividad que debe ser controlado de la mejor forma posible (GLOBERSON, 1985; FORTUIN, 1988; NEELY et al., 1996; NEELY et al., 1997; COYLE; BARDI; LANGLEY, 2002). Estos autores, también recomiendan que los indicadores de desempeño cuenten con valores cuantificables y fáciles de entender, sean prácticos y con escalas de medición apropiadas, mantengan su significancia a través del tiempo, tengan objetivos bien delimitados, consideren datos de entrada y salida, y sean productivos en relación a los costos de medición y análisis.

De la misma manera en que el SMD está formado por un conjunto de indicadores, éstos son descritos por una serie de atributos que definen las funciones, el momento y la forma de uso de cada indicador. Entre los principales atributos se tiene: nombre (debe ser claro y sin ambigüedades), objetivo o propósito (deben ser claros y relacionados a los objetivos organizacionales), meta (representando los objetivos organizacionales), ámbito (partes de la organización que participan en la evaluación), fórmula de cálculo (representa como se medirá el desempeño), frecuencia de medición y análisis (registro de la medida y elaboración de informes), frecuencia de revisión (reevaluación del diseño de indicadores), responsable de la medida (colectar datos y reportar mediciones) y responsable del análisis de resultados (NEELY et al., 1997; NEELY et al., 2002; GUNASEKARAN; 
PATEL; McGAUGHEY, 2004; LOHMAN; FORTUIN; WOUTERS, 2004). El Cuadro 1 presenta una lista con la descripción de los principales atributos.

Un SMD bien implementado debe contar con la capacidad de adquirir, almacenar, procesar, interpretar y distribuir informaciones sobre el desempeño de la organización, estimulando cambios gerenciales y promoviendo el aprendizaje organizacional (BITITCl; TURNER; BEGEMANN, 2000; BOURNE et al., 2000; GARENG0; BIAZZO; BITITCl, 2005). Esas funciones solo pueden ser alcanzadas cuando el SMD es correctamente estructurado en todas sus etapas de desarrollo: el diseño (traducción de las necesidades y objetivos de la empresa en un conjunto de indicadores apropiados), la implementación (requiere el soporte de sistemas de informaciones y el desarrollo de procedimientos para colectar, procesar y divulgar informaciones) y el uso del sistema de medición (requiere continuos procesos de revisión y ajustes) (BOURNE et al., 2000; HUDSON; SMART; BOURNE, 2000). Según Globerson (1985), un sistema de medición de desempeño puede ser orientado tanto para medir el desempeño de individuos como de organizaciones. Así también, puede ser orientado tanto para un nivel micro (áreas o departamentos), como para un nivel macro (cadenas de suministros), a través de la integración de varios niveles micro. En el desarrollo de SMD aplicado a cadenas de suministros existe la tendencia de utilizar indicadores que se concentran en mediciones internas de desempeño logístico, sin llegar a capturar el desempeño de la cadena de suministros como un todo. Esa concentración de mediciones delimita la capacidad de las métricas calculadas

Cuadro 1. Principales atributos.

\begin{tabular}{|c|c|}
\hline $\begin{array}{c}\text { Atributo de } \\
\text { indicadores }\end{array}$ & Descripción \\
\hline Nombre & Debe ser claro y sin ambigüedades \\
\hline $\begin{array}{c}\text { Objetivo o } \\
\text { propósito }\end{array}$ & $\begin{array}{c}\text { Debe ser claro y relacionado a los } \\
\text { objetivos organizacionales }\end{array}$ \\
\hline Meta & $\begin{array}{c}\text { Representa los objetivos } \\
\text { organizacionales }\end{array}$ \\
\hline Ámbito & $\begin{array}{c}\text { Partes de la organización que } \\
\text { participan en la evaluación }\end{array}$ \\
\hline $\begin{array}{c}\text { Fórmula de } \\
\text { cálculo }\end{array}$ & $\begin{array}{c}\text { Representa como se medirá } \\
\text { el desempeño }\end{array}$ \\
\hline $\begin{array}{c}\text { Frecuencia de } \\
\text { medición y análisis }\end{array}$ & $\begin{array}{c}\text { Registro de la medida y elaboración } \\
\text { de informes }\end{array}$ \\
\hline $\begin{array}{c}\text { Frecuencia de } \\
\text { revisión }\end{array}$ & $\begin{array}{c}\text { Reevaluación del diseño } \\
\text { de indicadores }\end{array}$ \\
\hline $\begin{array}{c}\text { Responsable de } \\
\text { la medida }\end{array}$ & $\begin{array}{c}\text { Colecta datos y reporta } \\
\text { mediciones }\end{array}$ \\
\hline $\begin{array}{c}\text { Responsable del } \\
\text { análisis de resultados }\end{array}$ & $\begin{array}{c}\text { Encargado de analizar los informes } \\
\text { provenientes del sistema }\end{array}$ \\
\hline
\end{tabular}

para determinar si el desempeño de las empresas ha mejorado o empeorado. (BEAMON, 1999; LAMBERT; POHLEN, 2001; SHEPHERD; GÜNTER, 2006). En ese sentido, Gunasekaran, Patel y Tirtiroglu (2001) señalan que las diferencias funcionales que los indicadores de desempeño tienen entre los niveles estratégico, táctico y operacional son confundidas en la práctica, especialmente cuando las mediciones provienen de fuentes externas, como es el caso de la evaluación del desempeño de proveedores dentro de una cadena de suministros (TATE; van DER VALK, 2008). De esta manera, Harrison, Lee y Neale (2003) advierten que el uso de indicadores inapropiados puede ser el mayor impedimento para la implementación de una gestión integrada y eficiente de la cadena de suministros.

la evaluación de proveedores es un eslabón importante en la secuencia de evaluaciones que determinan el desempeño de las cadenas de suministros. En ese contexto, los sistemas de medición de desempeño de proveedores (SMDP) tienen la finalidad de mejorar continuamente el desempeño de la empresa compradora a través del aprovechamiento de las capacidades de sus proveedores (WAGNER; JOHNSON, 2004). Asegurar el éxito de ese mecanismo de mejoría conjunta, exige que los objetivos de los proveedores y los de la empresa compradora estén alineados mediante la selección apropiada de indicadores SPE (TATE; van DER VALK, 2008). Otro factor importante para el éxito de los SMDP radica en el tipo de relacionamiento existente entre la empresa y sus proveedores. Los medios de comunicación entre ambas organizaciones deben asegurar que las medidas y metas esperadas de la empresa contratante sean transmitidas de tal manera que genere comprometimiento y colaboración por parte de los proveedores. Respecto a las condiciones de diseño de los SMDP, Tate y van Der Valk (2008) mencionan que los indicadores no deben considerar solo las características operacionales, recomendando que éstos sean en función de las competencias de: relacionamiento con la empresa, de producción y de entrega. En estas circunstancias, Coyle, Bardi y Langley (2002) aconsejan usar técnicas de benchmarking (estudio de las mejores prácticas en el mercado) para enriquecer la etapa de obtención de conocimientos necesarios para el diseño SMDP. Estudios referentes a las clases de indicadores que deben ser incluidos en los SMDP concluyen que organizaciones de buen desempeño tienden a dar menos importancia a mediciones respecto al precio y/o costo unitario, siendo que indicadores de calidad, confiabilidad en la entrega y performance 
del producto son las características que garantizan la coherencia de la evaluación con los objetivos estratégicos de la empresa (KAPLAN; NORTON, 1996; BEAMON, 1999; GUNASEKARAN; PATEL; McGAUGHEY, 2004; SHEPHERD; GÜNTER, 2006).

Una vez implementado el SMD, este debe ser constantemente actualizado (WISNER; FAWCETT, 1991; GHALAYINI; NOBLE, 1996). Los procesos de actualización buscan el alineamiento de los resultados del sistema de medición con la estrategia organizacional, donde, a través de continuas revisiones del sistema se mejore la calidad de decisiones tomadas a partir de las mediciones de desempeño obtenidas (BITITCl; TURNER; BEGEMANN, 2000; BOURNE et al., 2000; HUDSON; SMART; BOURNE, 2000). Tales actualizaciones deben eliminar los indicadores que dejaron de ser coherentes con las nuevas estrategias organizacionales.

\section{Método de investigación}

El método de investigación utilizado para diseñar el SMDP contó con cinco etapas. Estas etapas son descritas a seguir.

\subsection{Etapa 1: definición de los objetivos del SMDP}

La Etapa 1 define los objetivos de medición de la empresa con base en sus necesidades y en el tipo de decisiones que serán tomadas a partir de las mediciones obtenidas en el SMDP. Para tal fin, es primordial identificar, dentro de los procesos de compra, cuales son las principales características en la ejecución de la venta de productos o implementación de servicios, que determinan el nivel de desempeño del proveedor. Definir un concepto de desempeño esperado de proveedores, requiere un previo entendimiento de las diferentes corrientes culturales internas que rigen el comportamiento de la empresa, de tal manera que, se identifiquen puntos de convergencia entre las diferentes áreas o departamentos participantes en la evaluación de proveedores. Para llegar a esa convergencia fue utilizado un workshop multifuncional que reunió funcionarios del nivel estratégico. Ésta reunión contó con la participación de seis gerentes, cuatro del área de compras y con dos gerentes de las principales áreas internas atendidas por el departamento de Compras (clientes internos). La participación de los clientes internos es justificada por la existencia de determinados casos, en que el planeamiento de compras es totalmente realizado por ellos, dejando al departamento de Compras apenas con la responsabilidad de ejecutarlo y por la constante interacción con los proveedores durante las etapas de implementación de servicios y posentrega .

\subsection{Etapa 11: levantamiento de informaciones}

La Etapa 11 consistió en el levantamiento de informaciones externas (verificación de los indicadores y atributos SPE utilizados en el mercado por medio de un estudio de benchmarking) e internas (conocimiento de la realidad de la empresa CLAVE, mediciones formales e informales, así como los procesos organizacionales y herramientas de $\mathrm{Tl}$ que las soportan).

\subsubsection{Estudio de benchmarking}

El levantamiento de informaciones externas incluyó empresas que se caracterizaban por tener un perfil de compras semejante al utilizado en CLAVE o por ser consideradas como best-practice en el mercado. Parte de las informaciones requeridas se encontraban disponibles para el público en general (datos secundarios), en algunos casos fue preciso complementar informaciones por medio de entrevistas, contacto telefónico o e-mail (datos primarios). De esta manera se obtuvo un conjunto de 20 empresas representativas de diferentes industrias de producción y servicios, en las que se estudiaron y analizaron el uso de indicadores SPE, sub-indicadores y atributos (objetivos de medición, sistema de calificación, frecuencia de medición y de análisis y decisiones recomendadas a partir de los posibles resultados). Si bien existieron casos en que no fue posible completar todas las informaciones, ya sea por motivos de confidencialidad de información o porque la empresa analizada no contaba con la información solicitada, la masa de empresas estudiadas y las similitudes entre ellas permitieron identificar las técnicas y simplificaciones de medición de desempeño que están dando resultado en la práctica.

\subsubsection{Sistema de medición actual}

Paralelamente al levantamiento de informaciones externa (benchmarking), se realizó un análisis de la forma como era medido el desempeño de los proveedores en la empresa CLAVE. Tal estudio fue iniciado mediante un workshop con los gerentes y coordinadores más importantes del sector de compras de bienes y el sector de compras de servicios. El workshop contó con dos participantes 
por área y dos facilitadores encargados del estudio, siendo un total de seis personas las que colaboraron con el levantamiento de las informaciones internas del departamento de Compras.

A manera de detallar las particularidades de cada área, fueron realizadas entrevistas no estructuradas con cinco funcionarios del área de bienes y con otros cinco del área de servicios. Estas entrevistas contaron con la participación de los respectivos gerentes de área, dos coordinadores de mando medio de cada área y dos representantes de las áreasclientes más significativas para el departamento de Compras (tanto para bienes como para servicios). El conocimiento de los procesos y de las herramientas de Tl utilizadas fue mediante observaciones in loco, estas observaciones también fueron en forma particular para cada área de compras.

\subsection{Etapa 111 - definición de la propuesta de indicadores SPE}

Los autores de la investigación realizaron en la Etapa 111 un análisis triangular entre los resultados obtenidos con el benchmarking, el análisis de la realidad actual de la empresa y las necesidades identificadas en la Etapa 1 del método. Los resultados obtenidos fueron discutidos y analizados en dos workshops, uno con cada sector de compras (bienes y servicios). Tales workshops contó con la colaboración de los mismos funcionarios (diez) que participaron en la etapa del levantamiento de informaciones internas. El método de consenso fue utilizado para guiar las discusiones relacionadas a la definición del conjunto de indicadores y sus respectivos atributos, como son: subindicadores o drivers, objetivos, clase de medida utilizada (cuantitativa o cualitativa), metas y fórmulas de cálculo. De la misma forma también fueron definidos los responsables por las etapas de medición y análisis, y la forma como se llevarían a cabo las revisiones de los indicadores (importante etapa para acompañar el uso del sistema en el futuro).

\subsection{Etapa IV - validación}

Fuera de las continuas validaciones que permitieron la depuración de indicadores y atributos, fue preciso validar el conjunto de indicadores como un todo, explicando la función que cada atributo tiene en la descripción del desempeño del proveedor. Para eso, fue desarrollado, en una planilla electrónica, un prototipo que permitió modelar la alimentación de informaciones históricas de la empresa y los principales informes que resultarían a partir de las calificaciones de los indicadores.

Fueron realizadas dos reuniones de validación, la primera a nivel gerencial, con la participación de los cuatro gerentes de compras (dos por sector) y de los dos gerentes de las áreas-clientes (un por sector) que habían participado en los workshops de la Etapa 1. La segunda reunión, que fue realizada a nivel Directorio, tuvo la intención de discutir pequeños ajustes basados en fines estratégicos.

\subsection{Etapa $V$ - evaluación de alternativas de SI para la implementación del SMDP}

La Etapa V corresponde a la evaluación de diferentes sistemas de información disponibles en el mercado, con el objetivo de escoger la solución que brinde el mejor soporte para la implementación del SMDP diseñado. Esta etapa fue basada en las informaciones relativas al sistema ERP (Enterprise Resource Planning) y a las otras herramientas tecnológicas utilizadas en la gestión y control de los procesos de compras de CLAVE. Fueron identificados los datos de entrada (para los subindicadores) que se encontraban disponibles en las herramientas de información utilizadas. Estos datos fueron descritos a partir de su localización y de las características del registro y acceso a las informaciones relativas a ellos. A partir de esa información se determinaron que requisitos básicos de comunicación deberían cumplir los sistemas de información candidatos. La decisión fue reducida a cuatro opciones, tres relativas a una solución externa y una a la posibilidad de desarrollar un sistema in-house (a cargo del propio departamento de $\mathrm{Tl}$ de (LAVE). Fueron realizadas dos reuniones con cada candidato, una para presentar el diseño y requerimientos de implementación del SMDP y otra para recibir las respectivas propuestas.

La Figura 1 presenta una síntesis de las cinco etapas de método de investigación.

\section{Diseño y análisis del SMDP}

Siguiendo la lógica definida en el método de investigación, esta sección presenta la descripción y análisis de los resultados obtenidos en el estudio.

\subsection{Etapa 1: definición de los objetivos del SMDP}

Con base en los requerimientos del departamento de Compras y de sus principales clientes internos, se 


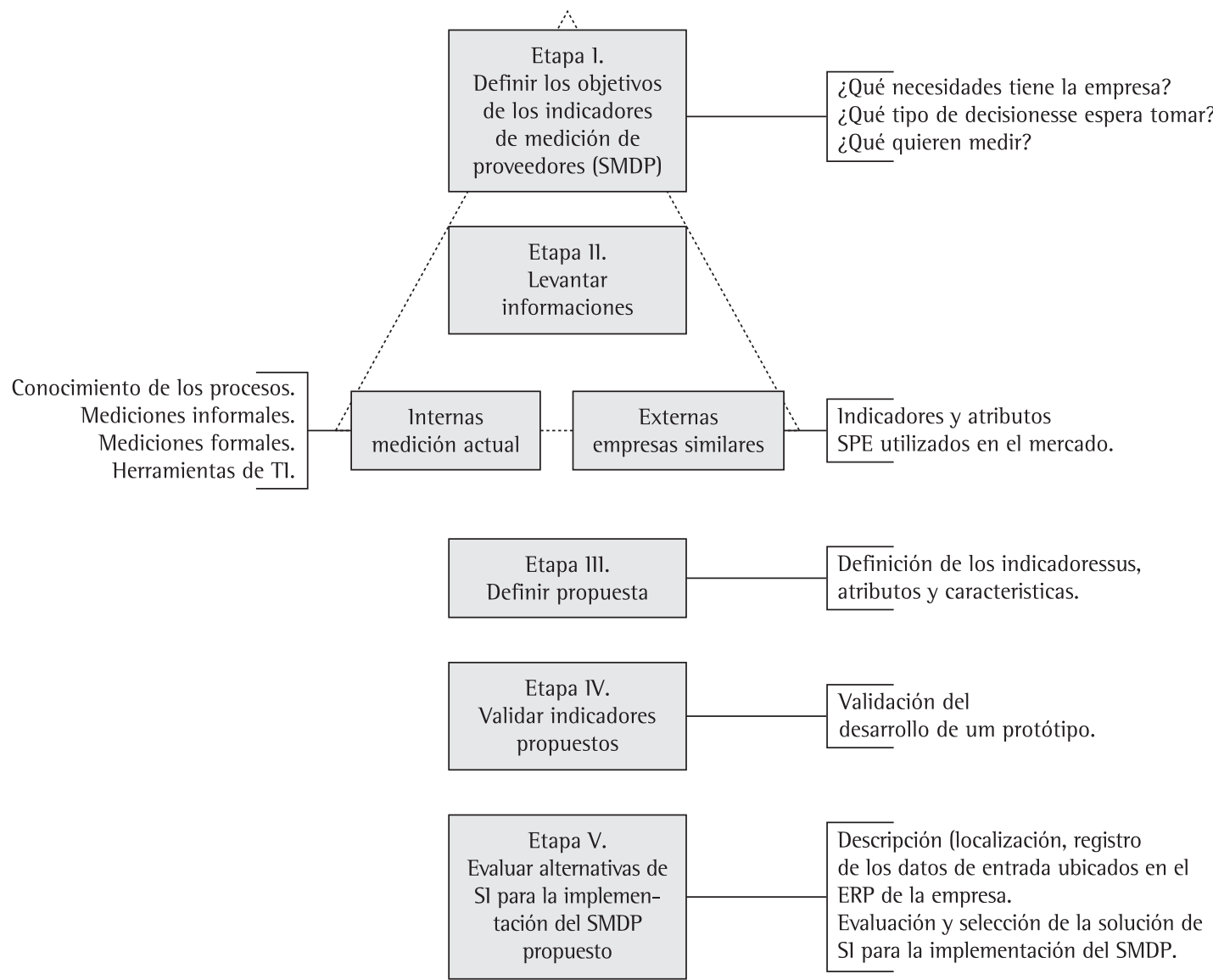

Figura 1. Etapas del método de investigación.

definieron las siguientes necesidades (resumen en el Cuadro 2):

- Existe la necesidad de disminuir la base de proveedores, de tal manera que esta incluya apenas proveedores de confiabilidad asegurada;

- Es necesario clasificar a los proveedores en función de su desempeño, identificando aquellos que cumplieron correctamente con las expectativas de la empresa, diferenciando cuales cuentan con las características de un "aliado estratégico" e identificando los que cumplen con los requerimientos necesarios para atender compras de bienes o servicios de alto impacto para la empresa CLAVE;

- Es preciso establecer un lenguaje de comunicación estructurado para dar a conocer a los proveedores cual es su nivel de desempeño en relación a las expectativas de la empresa, incentivando la participación del mismoen el proceso de mejora continua de los procesos productivos de la empresa.
Con base en tales necesidades se definió que los indicadores SPE soportarían las siguientes decisiones:

- Retirar los proveedores con desempeños no aceptables, es decir, identificar que proveedores no deben ser convocados para nuevas cotizaciones o licitaciones;

- Realizar llamadas de atención y suspensiones de aquellos proveedores que den indicios de decaimiento en su desempeño;

- Reconocer públicamente y privilegiar a los proveedores de mejor desempeño;

- Formar alianzas estratégicas con los proveedores que están en la misma línea de trabajo y demuestren que son capaces y confiables;

- Congelar el número de cotizaciones de los proveedores que demuestren un aumento desproporcionado en la dependencia económica de la empresa CLAVE.

Las características de las mediciones correspondientes a tales necesidades y decisiones esperadas son: 
Cuadro 2. Necesidades y decisiones de la empresa en el SMDP.

\begin{tabular}{|c|c|}
\hline Necesidades de la empresa & Decisiones que os indicadores soportarian \\
\hline $\begin{array}{l}\text { Disminuir la base de proveedores, de tal manera que esta incluya apenas } \\
\text { proveedores de confiabilidad asegurada. }\end{array}$ & $\begin{array}{l}\text { Retirar los proveedores con desempeños no aceptables, } \\
\text { identificando que proveedores no deben ser convocados para } \\
\text { nuevas cotizaciones o licitaciones. }\end{array}$ \\
\hline \multirow{2}{*}{$\begin{array}{l}\text { Clasificar los proveedores en función de su desempeño, identificando } \\
\text { proveedores que cumplieron correctamente con las expectativas de la } \\
\text { empresa, proveedores que cuentan con las características de un "aliado } \\
\text { estratégico" y proveedores que cumplen con los requerimientos necesarios } \\
\text { para atender compras de bienes o servicios de alto impacto para Clave. }\end{array}$} & $\begin{array}{l}\text { Formar alianzas estratégicas con los proveedores que están } \\
\text { en la misma línea de trabajo de Clave y demuestren que son } \\
\text { capaces y confiables. }\end{array}$ \\
\hline & $\begin{array}{l}\text { Congelar el número de cotizaciones de los proveedores que } \\
\text { demuestren un aumento desproporcionado en la dependencia } \\
\text { económica de la empresa Clave. }\end{array}$ \\
\hline \multirow{2}{*}{$\begin{array}{l}\text { Establecer un lenguaje de comunicación estructurado para dar a conocer } \\
\text { a los proveedores cual es su nivel de desempeño en relación a las } \\
\text { expectativas de la empresa, incentivando la participación del proveedor en } \\
\text { el proceso de mejora continua de los procesos productivos de la empresa. }\end{array}$} & $\begin{array}{l}\text { Realizar llamadas de atención y suspensiones de aquellos } \\
\text { proveedores que den indicios de decaimiento en su desempeño. }\end{array}$ \\
\hline & $\begin{array}{l}\text { Reconocer públicamente y privilegiar a los proveedores de } \\
\text { mejor desempeño. }\end{array}$ \\
\hline
\end{tabular}

- Las mediciones del desempeño de los proveedores serán realizadas solo durante las etapas de poscompra, es decir, desde que se concreta la negociación con el proveedor seleccionado hasta la entrega del producto y durante los servicios posventa;

- Aspectos relacionados al estado financiero de la empresa proveedora deben ser considerados en la evaluación de su capacidad para mantener un suministro estable;

- Aspectos relacionados a la calidad y entrega de los productos y servicios ofertados son de importancia primordial para la medición del desempeño de los proveedores.

\subsection{Etapa 11: levantamiento de informaciones}

Esta etapa es dividida en dos tipos de informaciones: informaciones de origen externo, provenientes de un estudio de benchmarking e informaciones de origen interno, provenientes del estudio de la realidad de la empresa.

\subsubsection{Estudio de benchmarking}

Los indicadores y subindicadores recolectados fueron inicialmente organizados de acuerdo a clasificaciones y agrupamientos encontrados con mayor frecuencia entre las empresas estudiadas.

El estudio mostró que los indicadores de Calidad y Logística/Entrega están presentes en la mayoría de evaluaciones analizadas. Los aspectos evaluados con mayor frecuencia en la calidad son relacionados a los subindicadores: cumplimiento de las especificaciones del producto/servicio (85\%) y calidad en los procesos de producción (50\%). Los aspectos más evaluados en relación a la logística/ entrega son: divergencias en la fecha de entrega (95\%) y en la cantidad entregada (85\%). Existe un cierto rechazo para evaluar el desempeño de los proveedores en función de aspectos económicos como precio (40\%) y costos (15\%). Si bien existió un número considerable de empresas que utilizaba el indicador precio, la gran mayoría concordó que el precio del producto o servicio ofrecido no da una posición diferencial entre lo que significa buen y mal desempeño del proveedor. Alternativamente, pocas empresas de mayor experiencia en indicadores SPE utilizan conceptos de costos para identificar el impacto económico del desempeño de sus proveedores. Indicadores relativos al relacionamiento estratégico con los proveedores son menos encontrados en el mercado, indicando que existe una incapacidad práctica para evaluar los niveles de colaboración entre clientes y proveedores. Tal fenómeno se debe a la nebulosidad existente en la definición del significado de "aliado" y a la dificultad para lidiar con la subjetividad inherente en su medición. Otros indicadores referentes al nivel de flexibilidad y a la capacidad de gerenciamiento organizacional son también poco utilizados en la práctica, aún cuando la organización reconoce su impacto en las decisiones tomadas.

En relación a los métodos de calificación, el estudio muestra que aunque sea recomendado disminuir el número de indicadores subjetivos, en la práctica, resulta difícil prescindir de la percepción del evaluador, siendo que tanto el uso de índices cuantitativos como el uso de conceptos cualitativos tienen el mismo porcentaje de uso (58\%). El uso de listas de ocurrencias (32\%) o calificación a partir de la contabilidad de disconformidades es otra forma de medición que, a pesar de precisar de la observación humana y de no ser totalmente automática, permite que la percepción del evaluador tome valores cuantificables y menos subjetivos. Los resultados muestran la necesidad de diferenciar el impacto que cada subindicador tiene sobre el 
concepto de desempeño del proveedor, siendo que los métodos de medición ponderada (58\%) son más utilizados que los métodos de medición pura (11\%).

Los objetivos de medición analizados fueron clasificados según su envergadura, pudiendo ser objetivos relacionados solo al departamento de Compras u objetivos que consideran el impacto de las acciones del proveedor en otras áreas de la empresa. Este último tipo de objetivo busca implementar un sistema de mejora continua con los proveedores de más impacto en los procesos de la empresa. Los objetivos más citados relacionados a compras fueron: escoger proveedores nuevos (17\%), clasificar proveedores (50\%), disminuir el número de proveedores (17\%) y desarrollar proveedores “aliados" (58\%). El primer objetivo fue desconsiderado por no ser coherente con los requerimientos de la empresa en evaluar solo etapas de poscompra. Resulta interesante, como en la mayoría de empresas se reconoce la necesidad de mejorar la relación de alianza estratégica con los proveedores, sin embargo, la difícil determinación y medición de tales características hace que el uso de indicadores específicos para ello sea desconsiderado. Entre las decisiones más utilizadas a partir de los resultados de indicadores tenemos: Suspensión del proveedor (29\%), aceptación de proveedores (21\%), substitución del proveedor (21\%), congelamiento del número de ítems cotizados (7\%), solicitar un planeamiento de acciones correctivas (36\%), reconocimientos de buen desempeño (64\%) y de calidad asegurada (14\%). Estos resultados son coherentes con las necesidades definidas en la empresa CLAVE.

\subsubsection{Sistema de medición actual}

Inicialmente fue identificado que la empresa CLAVE no contaba con un registro formal de mediciones que describiera sistemáticamente el comportamiento y características de los proveedores inscritos en el banco de datos de la empresa. No obstante, el sector de compras de servicios utilizaba una serie de mediciones caracterizadas por ser subjetivas y registradas en formato físico (papel). Tal procedimiento fue considerado como una medición informal por no seguir una estructura predeterminada de medición y análisis, además de ser basado en cuestionarios específicos para cada tipo de cliente. A pesar de la intuición ganada a partir de esas evaluaciones esporádicas en el sector de compras de servicios, se observó que, en general, el personal de compras no era capaz de identificar ni clasificar a sus proveedores en buenos, regulares y/o malos. La poca coherencia en las informaciones recolectadas por medio de los cuestionarios llevaba al personal de servicios a tomar decisiones de la misma forma que en el sector de bienes, empíricamente y con base en la experiencia y memoria de los compradores.

Entre las particularizaciones existentes en cada sector de compras se identificó que el sector de bienes realizaba todos los procesos de compras a través del sistema ERP de la empresa, mientras que el sector de compras de servicios utilizaba un sistema paralelo creado especialmente para dar soporte a las diferentes clases y procedimientos de servicios. Dos clases de servicios fueron identificados: servicios continuos (que trabaja con un sistema de renovación periódica de contratos) y servicios no continuos (contratos de periodos cortos que tienen una fecha de inicio y término específica). No obstante, dentro de cada clase existe una infinidad de tipos de servicios, que van desde servicios técnicos hasta tercerizaciones de funciones de limpieza y seguridad. En general, ambos sectores de compras tienen comportamientos diferenciados, donde los procesos de compras, evaluación de proveedores, contacto con los clientes internos y tomada de decisiones, son basados en una subcultura organizacional que, aunque sea alineada con los objetivos de la gerencia estratégica, implica una complejidad mayor que exige de evaluaciones diferenciadas para cada sector.

\subsection{Etapa 111: definición de la propuesta de indicadores SPE}

La propuesta de indicadores SPE nació a partir de la triangulación de los resultados del estudio de benchmarking, del levantamiento de informaciones de la empresa y del análisis de sus necesidades.

\subsubsection{Indicadores}

Los indicadores con mayor incidencia que satisfacían uno o más requerimientos de la empresa fueron incluidos en la propuesta. Inicialmente, el estudio de las necesidades y requerimientos de la empresa indicó que los indicadores de calidad y entrega formarían parte del SMDP. Posteriormente, a raíz del análisis más profundo de las decisiones estratégicas y operacionales de la empresa, se percibió la necesidad de incluir indicadores relativos al nivel de confiabilidad necesaria para poder considerar a los proveedores como "aliados estratégicos", al nivel de flexibilidad ofrecida para atender cualquier tipo de variación durante la 
ejecución de la compra, y a la robustez o capacidad financiera necesaria para soportar los servicios y productos comercializados sin crear dependencias económicas con la empresa CLAVE.

Debido a las particularidades de ambos sectores de compras (bienes y servicios) y a la variedad de tipos de proveedores con los que estos trabajaban, los autores del estudio establecieron la premisa de definir pocos indicadores, que sean relevantes y satisfagan las necesidades de la empresa en forma homogénea y simple. De esta manera, se definieron los indicadores de Calidad, Entrega, Flexibilidad, y Riesgo Financiero para ambos sectores, estableciéndose que la descripción general del desempeño de los proveedores sería basada en la combinación equitativa de los cuatro indicadores. De esa forma se evitaría el uso de ponderaciones que particularice cada sector y reste significancia gerencial al SMDP.

Las características de confiabilidad necesaria para que los proveedores sean considerados "aliados estratégicos" se obtuvieron a partir de la combinación de los cuatro indicadores definidos. Tal explicación es detallada más adelante, en la sección de Análisis de las Mediciones.

\subsubsection{Subindicadores o drivers}

Fueron escogidos subindicadores que sean coherentes con la cultura de la empresa y con la complejidad deseada en los procesos de medición y análisis. En ese sentido, las diferencias entre los sectores de compra de bienes y servicios son tratadas individualmente, definiendo, siempre que sea necesario, subindicadores específicos para cada sector. Las diferencias entre ambos sectores fueron concentradas únicamente en los indicadores de calidad y entrega, los indicadores de flexibilidad $\mathrm{y}$ riesgo financiero permanecieron iguales para todo el departamento de Compras. De la misma manera, las dos subclases de servicios (continuos y no continuos) también fueron diferenciadas en la elección de subindicadores, siendo que tales diferencias se concentran en la definición de los pesos de importancia de los subindicadores que conforman los indicadores de calidad, entrega y riesgo financiero. A continuación son listados los subindicadores que conforman cada indicador, entre paréntesis se indican los sectores y subclases donde estos actúan:

- Calidad: Cumplimiento de las especificaciones del producto o servicio. Disponibilidad de atendimiento y asistencia técnica (bienes y servicios). Calidad en los procesos de implementación y Seguridad (servicios);
- Entrega: Divergencia en la fecha de entrega y Comunicación de los cambios en la fecha de entrada real (bienes y servicios no continuos). Divergencias en la cantidad entregada. Errores en la factura y embalaje adecuado (bienes);

- Flexibilidad: Indicador libre que permite el registro de subindicadores dinámicos. Nuevos requerimientos de flexibilidad son creados cada vez que la empresa solicite alguna modificación de entrega, calidad, pago, etc;

- Riesgo Financiero: Riesgo financiero del proveedor y Grado de dependencia (Evalúa cuál es la participación de la empresa CLAVE en la totalidad de ventas del proveedor).

Algunos subindicadores como: disponibilidad de atendimiento, seguridad, errores en la factura y embalaje adecuado, no tuvieron una frecuencia de uso significativa en el estudio de benchmarking. Sin embargo, éstos fueron considerados no SMDP por constatarse que tales características del proveedor describen aspectos importantes para la eficiencia productiva del departamento de Compras y para el cumplimiento de los objetivos de aumentar la satisfacción del cliente interno.

El Cuadro 3 presenta los principales atributos considerados para la descripción de los indicadores y sub-indicadores incluidos en el SMDP.

La Tabla 1 son mostrados los subindicadores y la importancia relativa (su peso) con que cada uno conforma los indicadores designados para los diferentes sectores: bienes (B), servicios continuos (SC) y servicios no continuos ( $\mathrm{SnC}$ ). Es así que el valor numérico otorgado al indicador es dado por el sumatorio de las calificaciones ponderadas de cada subindicador.

\subsubsection{Atributos}

Esta sección presenta los principales atributos considerados para la descripción de los indicadores y subindicadores incluidos en el SMDP. La elección y descripción de tales atributos fue basada tanto en la literatura académica como en las necesidades de la empresa.

- Objetivos, Responsabilidades y Frecuencias: Estos atributos fueron definidos para cada subindicador. Las descripciones fueron claras y concisas, así como la atribución de responsabilidades fue considerando el nivel de interacción con el proveedor. Cuando los subindicadores de un mismo indicador son medidos con diferentes frecuencias, solo son computados aquellos drivers que tienen valor.

- Pesos y metas: A partir de la experiencia de los compradores y clientes internos, fueron definidos, para cada subindicador, diferentes 
pesos o importancia en la conformación del indicador correspondiente. El mismo criterio fue utilizado para la definición de las metas, cuyos valores dan origen a los límites que definen las diferentes clasificaciones de los proveedores. La determinación de los pesos y metas de todos los subindicadores fueron posteriormente validados mediante el uso de datos históricos.

Cuadro 3. Atributos de indicadores y sub-indicadores del SMDP.

\begin{tabular}{|c|c|}
\hline $\begin{array}{l}\text { Objetivos, } \\
\text { responsabilidades } \\
\text { y frecuencias }\end{array}$ & $\begin{array}{l}\text { Estos atributos fueron definidos para cada sub-indicador. Las descripciones fueron claras y concisas, así como la } \\
\text { atribución de responsabilidades fue considerando el nivel de interacción con el proveedor. Cuando los sub-indicadores } \\
\text { de un mismo indicador son medidos con diferentes frecuencias solo son computados aquellos que tienen valor. }\end{array}$ \\
\hline Pesos y metas & $\begin{array}{l}\text { A partir de la experiencia de los compradores y clientes internos, a través del método de consenso fueron definidos, } \\
\text { para cada sub-indicador, diferentes pesos o importancia en la conformación del indicador correspondiente. El mismo } \\
\text { criterio fue utilizado para la definición de las metas, cuyos valores dan origen a los límites que definen las diferentes } \\
\text { clasificaciones de los proveedores. La determinación de los pesos y metas de todos los sub-indicadores fueron } \\
\text { posteriormente validados con auxilio del uso de datos históricos. }\end{array}$ \\
\hline Calificación & $\begin{array}{l}\text { Se definió una escala de calificación de } 3 \text { valores ( } 0,2 \text { ó 4), donde para peores desempeños, mayor será el valor } \\
\text { calificado. El valor nulo identifica el cumplimiento esperado de desempeño. Este sistema disminuye la subjetividad } \\
\text { en las calificaciones cualitativas. El uso de descripciones de ocurrencias que tipifican cada valor posible }(0,2 \text { ó } 4) \text { se } \\
\text { evita que el evaluador califique por afinidades personales. Los valores cuantitativos como índices porcentuales son } \\
\text { convertidos a la escala utilizada por medio de tablas de metas predefinidas. }\end{array}$ \\
\hline Revisiones & $\begin{array}{l}\text { Por tratarse de una empresa que necesita administrar una amplia diversidad de proveedores, y que no contaba con } \\
\text { experiencia en SMDP, se definió que durante el primer año de uso, las revisiones serian realizadas trimestralmente. } \\
\text { Conforme los usuarios vayan ganando experiencia y los requerimientos de ajustes en interfaces, procesos y/o métodos } \\
\text { del SMDP se hayan satisfecho, dicha periodicidad será semestralmente. Una vez que el uso del SMDP sea estable, las } \\
\text { revisiones se concentrarán en la validación de la congruencia entre los indicadores y las mudanzas en la estrategia } \\
\text { organizacional. Tales revisiones serán realizadas a cada año. En todas las revisiones fue recomendado dar mayor } \\
\text { atención al ajuste de los pesos y metas definidos para cada sub-indicador. }\end{array}$ \\
\hline
\end{tabular}

Tabla 1. Indicadores y sub-indicadores del SMDP.

\begin{tabular}{|c|c|c|c|c|c|c|}
\hline \multirow{3}{*}{ Indicador } & \multirow{3}{*}{ Sub-indicador } & \multirow{3}{*}{ Descripción } & \multirow{3}{*}{$\begin{array}{l}\text { Área de } \\
\text { compras }\end{array}$} & \multicolumn{3}{|c|}{ Peso } \\
\hline & & & & \multicolumn{2}{|c|}{ Serv. } & \multirow{2}{*}{$\begin{array}{c}\text { Bienes } \\
(\%)\end{array}$} \\
\hline & & & & $\begin{array}{l}\mathrm{SC} \\
(\%)\end{array}$ & $\begin{array}{l}\mathrm{SnC} \\
(\%)\end{array}$ & \\
\hline \multirow{4}{*}{$C_{p}=0.8[C 1]+0.2[C 2]$} & $\begin{array}{c}\text { Cumplimiento de las } \\
\text { especificaciones del producto } \\
\text { o servicio (C1) }\end{array}$ & $\begin{array}{l}\text { El producto corresponde a las } \\
\text { especificaciones solicitadas }\end{array}$ & $\mathrm{B}, \mathrm{SnC}$ & 0 & 50 & 80 \\
\hline & $\begin{array}{l}\text { Disponibilidad de atendimiento } \\
\text { y asistencia técnica (C2) }\end{array}$ & $\begin{array}{l}\text { Capacidad de atender dudas y/o } \\
\text { resolver problemas pos-venda }\end{array}$ & $\mathrm{B}, \mathrm{SC}, \mathrm{SnC}$ & 10 & 10 & 20 \\
\hline & $\begin{array}{l}\text { Calidad en los procesos de } \\
\text { implementación (C3) }\end{array}$ & $\begin{array}{l}\text { Las especificaciones de calidad } \\
\text { en mano de obra, insumos, } \\
\text { organización y comunicación del } \\
\text { estatus del proyecto son atendidas } \\
\text { durante el proyecto }\end{array}$ & $\mathrm{SC}, \mathrm{SnC}$ & 80 & 30 & 0 \\
\hline & Seguridad (C4) & $\begin{array}{c}\text { El proveedor sigue procedimientos } \\
\text { de seguridad exigidos }\end{array}$ & $\mathrm{SC}, \mathrm{SnC}$ & 10 & 10 & 0 \\
\hline \multirow{5}{*}{$\begin{array}{c}E_{p}=0.4[E 1]+0.1[E 2]+ \\
0.1[E 3]+0.2[E 4]+0.2[E 5]\end{array}$} & $\begin{array}{c}\text { Divergencia en la fecha de } \\
\text { entrega (E1) }\end{array}$ & $\begin{array}{l}\text { Entregas fuera del plazo } \\
\text { especificado }\end{array}$ & $\mathrm{B}, \mathrm{SnC}$ & 0 & 80 & 40 \\
\hline & $\begin{array}{c}\text { Comunicación de los cambios en } \\
\text { la fecha de entrada real (E2) }\end{array}$ & $\begin{array}{l}\text { Manutención de los cambios de } \\
\text { fechas de entrega }\end{array}$ & $\mathrm{B}, \mathrm{SnC}$ & 0 & 20 & 20 \\
\hline & $\begin{array}{l}\text { Divergencias en la cantidad } \\
\text { entregada (E3) }\end{array}$ & Entregas parciales o incompletas & B & 0 & 0 & 20 \\
\hline & Errores en la factura (E4) & Errores en el llenado de factura & $\mathrm{B}$ & 0 & 0 & 10 \\
\hline & Embalaje adecuado (E5) & $\begin{array}{l}\text { Productos damnificados, embalaje } \\
\text { quebrada, rótulos equivocados, etc. }\end{array}$ & B & 0 & 0 & 10 \\
\hline $\begin{array}{l}\text { Flexibilidad } \\
\qquad F_{p}=F 1\end{array}$ & $\begin{array}{l}\text { Indicador libre que permite } \\
\text { el registro de sub-indicadores } \\
\text { dinámicos. }(\mathrm{F} 1)\end{array}$ & $\begin{array}{c}\text { Tiempo necesario para reaccionar } \\
\text { a cambios en precio, entrega, } \\
\text { pagos, etc. }\end{array}$ & $\mathrm{B}, \mathrm{SC}, \mathrm{SnC}$ & 100 & 100 & 100 \\
\hline $\begin{array}{l}\text { Riesgo Financiero } \\
\qquad \mathrm{R}_{\mathrm{SnC}}=\mathrm{RF} 1\end{array}$ & $\begin{array}{l}\text { Riesgo financiero del proveedor } \\
\text { (RF1) }\end{array}$ & $\begin{array}{l}\text { Evaluación de la salud financiera } \\
\text { del proveedor }\end{array}$ & SC & 100 & 0 & 0 \\
\hline
\end{tabular}

${ }^{*}$ B: bienes; SC: servicios continuos; SnC: servicios no continuos. 
- Calificación: Se definió una escala de calificación de 3 valores (0, 2 ó 4), donde peores desempeños obtendrán mayor valor calificado. El valor nulo identifica el cumplimiento esperado de desempeño. Este sistema disminuye la subjetividad en las calificaciones cualitativas. El uso de descripciones de ocurrencias que tipifican cada valor posible ( 0,2 ó 4) evita que el evaluador califique por afinidades personales. Los valores cuantitativos como índices porcentuales son convertidos a la escala utilizada por medio de tablas de metas predefinidas.

- Revisiones: Por tratarse de una empresa que necesita administrar una amplia diversidad de proveedores, y que no contaba con experiencia en SMDP, se definió que durante el primer año de uso, las revisiones serían realizadas trimestralmente. Conforme los usuarios vayan ganando experiencia y los requerimientos de ajustes en interfaces, procesos y/o métodos del SMDP se hayan satisfecho, dicha periodicidad será semestralmente. Una vez que el uso del SMDP sea estable, las revisiones se concentrarán en la validación de la congruencia entre los indicadores y las mudanzas en la estrategia organizacional. Tales revisiones serán realizadas a cada año. En todas las revisiones es recomendado dar mayor atención al ajuste de los pesos y metas definidos para cada subindicador.

\subsubsection{Ejemplo para los atributos del indicador Calidad}

A manera de ilustrar el proceso de evaluación de proveedores, se presenta la descripción del indicador de Calidad diseñado para el sector de compra de bienes.

El Cuadro 4 muestra la descripción de los atributos que caracterizan al primer driver del indicador Calidad [C1]. El conjunto de atributos delinea la funcionalidad, el momento y la forma en que las mediciones serán realizadas, siendo que cada atributo está alineado con el objetivo del subindicador y con los objetivos del propio indicador Calidad.

\subsubsection{Análisis de las mediciones}

Considerando que las puntuaciones agregadas de los indicadores pueden tomar valores fraccionarios entre 0,2 y 4 ; fueron definidos una escala de cuatro posibles niveles de calificación para describir el desempeño del proveedor en cada indicador (segunda columna de la Tabla 2). Cada nivel es identificado gráficamente por medio de un color característico que facilite su identificación.

La combinación de los niveles de calificación de los cuatro indicadores del SMDP describe el desempeño final del proveedor. Éste es catalogado en seis posibles áreas de desempeño: mejor proveedor, aliado estratégico, desempeño aceptable, área de observación, área de advertencia y desaprobado (Cuadro 5). Cada área de desempeño es identificada por símbolos gráficos (segunda columna) para facilitar el análisis posterior de las informaciones.

El área de desempeño denominada "aliado estratégico" exige que el proveedor, además de cumplir con el mejor desempeño esperado, presente rasgos proactivos en relación a ofrecer el mejor nivel de flexibilidad, que en principio no es obligado a cumplir.

Las acciones sugeridas (tercera columna) corresponden a las decisiones identificadas en la Etapa 1 del método.

Cuadro 4. Descripción de sub-indicadores.

\begin{tabular}{|c|c|c|}
\hline \multicolumn{3}{|c|}{ Indicador: calidad } \\
\hline \multicolumn{3}{|c|}{ Sub-indicador: cumplimiento de las especificaciones del producto [C1] } \\
\hline Objetivo & Medir el grado de cumplimiento de las espe & técnicas del producto (durante la entrega). \\
\hline Responsable por la medida & $\begin{array}{l}\text { Cliente interno: Cuando la inconformidad es } \\
\text { Gestor de compra: Cuando la inconformidac }\end{array}$ & $\begin{array}{l}\text { al final del proceso de compra. } \\
\text { da durante la entrega (almacén). }\end{array}$ \\
\hline Frecuencia medición & Cada vez que exista una inconformidad & \\
\hline Calificación & $\frac{\text { Items no conformes en la } O C}{\text { Número total de items de la OC (\%) }}$ & $\begin{array}{l}\text { Transformación de valor porcentual a escala } \\
\qquad \begin{array}{l}>10 \% \rightarrow 4 \\
>=2,5 \% \mathrm{y}<=10 \% \rightarrow 2 \\
<2,5 \% \rightarrow 0\end{array}\end{array}$ \\
\hline Calculo métrica mensual & {$[C 1]=\frac{\text { (Suma de las pur }}{\text { (Nume }}$} & $\frac{p r \text { OC del proveedor en el mes) }}{\text { proveedor en el mes) }}$ \\
\hline Revisiones & Trimestralmente & \\
\hline
\end{tabular}

*0C: orden de compra. 


\subsection{Etapa IV: validación}

El prototipo de los indicadores SPE seleccionados fue desarrollado para modelar y validar las funciones de cada indicador en ambos sectores de compra. Con el uso de datos históricos (3 meses) de la empresa, se simuló tanto el ingreso de información manual (caso de servicios) como de información proveniente del sistema ERP de la empresa. Fueron modelados dos clases de informes: agregado (nivel directorio) y detallado (nivel gerencial). Se indicó que la implementación real de los indicadores permitiría realizar filtros especiales, además de permitir la creación de escenarios a través de modificaciones temporarias en los pesos de indicadores. En las reuniones de nivel gerencial y directorio se reconoció que los usuarios necesitan conocer mejor a sus proveedores para definir pesos y metas más exactos.

\subsection{Etapa V: evaluación de alternativas de Sl para la implementación del SMDP}

El departamento de Compras utiliza un sistema ERP que controla e integra no solo las operaciones del departamento, sino también, las operaciones de todas las demás áreas y sucursales

Tabla 2. Escala de calificaciones.

\begin{tabular}{|c|c|c|}
\hline Intervalos calificación & Nivel & Símbolo \\
\hline 0-0.99 & 1 & Verde \\
\hline $1-1.99$ & 2 & Amarillo \\
\hline $2-2.9$ & 3 & Anaranjado \\
\hline $3-4$ & 4 & Rojo \\
\hline
\end{tabular}

de la empresa. Esta plataforma constituye el principal requerimiento de comunicación que el Sl que soporte la implementación del SMDP debe cumplir. Además de contar con la capacidad de leer e interpretar las informaciones del ERP, el SI debe ser capaz de comunicarse con dos sistemas creados específicamente para tratar contratos de servicios y operaciones de exportación. Los principales datos de entrada necesarios para o SMDP estaban concentrados en el sistema ERP y eran relacionados al indicador Entrega (fecha de entrega y cantidad entregada). No obstante, informaciones secundarias que permiten el seguimiento e identificación del desempeño de los proveedores, como son: código de la orden de compra, código del producto, cliente interno o solicitante de la compra, comprador, alteraciones de las fechas de entrega planeadas, etc., precisaban de la integración y comunicación con los sistemas de contratos y exportación. Los datos de entrada de los demás subindicadores no formaban parte de ningún sistema, por lo que era necesario que el SI base para la implementación del SMDP cree formularios y cuente con la capacidad de registrar la información en las base de datos actualmente utilizadas.

Ese contexto de desintegración de datosy manejo de diferentes plataformas de información hace que, el número de adecuaciones necesarios para que un sistema comercial estructurado (sin ser o propio ERP) atienda los requerimientos de integridad de la información, sea una solución excesivamente costosa. Esta problemática fue observada por los proveedores de sistemas de evaluación de desempeño desarrollados en plataformas de ERP diferentes, reconociendo la incapacidad de presentar soluciones económicamente competitivas. El mismo tipo de solución comercializada por los proveedores

Cuadro 5. Descripción del desempeño final del proveedor.

\begin{tabular}{|c|c|c|}
\hline Nivel alcanzado & Estado & Acción sugerida \\
\hline Todos los indicadores (menos flexibilidad) son calificados en el nivel 1 & छ્वे छी & $\begin{array}{l}\text { Proveedor } 5 \text { estrellas } \\
\text { Fomentar participación }\end{array}$ \\
\hline Todos los indicadores, incluyendo el indicador flexibilidad, son calificados en el nivel 1 & sis & Proveedor aliado estratégico \\
\hline $\begin{array}{l}\text { Por lo menos tres indicadores calificados en el nivel } 1 \text {. Solo pueden existir hasta dos } \\
\text { indicadores en el nivel } 2\end{array}$ & है। & $\begin{array}{l}\text { Proveedor con desempeño aceptable } \\
\text { Comunicar puntos de mejora }\end{array}$ \\
\hline Posee como máximo un indicador en el nivel 3 y ningún indicador en el nivel 4 & 㝖 & $\begin{array}{l}\text { Proveedor en el área de observación } \\
\text { Exigir plan de acciones correctivas }\end{array}$ \\
\hline $\begin{array}{l}\text { Puede tener hasta tres indicadores en el nivel } 3 \text { o hasta dos indicadores en el nivel } 4 \text {, } \\
\text { sin poder tener ambos niveles en una sola combinación }\end{array}$ & Why & $\begin{array}{l}\text { Proveedor en el área de advertencia } \\
\text { Disminución del número de licitaciones }\end{array}$ \\
\hline Posee más de tres indicadores en el nivel 3 o más de dos indicadores en el nivel 4 & 鲜 & $\begin{array}{l}\text { Proveedor desaprobado } \\
\text { Suspensión, búsqueda de proveedores } \\
\text { sustitutos }\end{array}$ \\
\hline
\end{tabular}


del ERP usado en la empresa no satisfacía las diversas necesidades de la empresa, siendo que su utilización significaría el sacrificio de informaciones importantes para la evaluación de los proveedores y para el cumplimiento y control de objetivos estratégicos de la empresa. Particularizaciones de dicha solución acarrearía un aumento significativo de los costos de implementación. Fue decidido que el propio departamento de $\mathrm{Tl}$ de la empresa llevaría a cabo la implementación del sistema, este contaría con el apoyo de una empresa especialista en desarrollo de sistemas de información y de los propios diseñadores del sistema.

\section{Conclusión}

A partir del estudio de una empresa multinacional de grande porte, este artículo presenta las etapas elaboradas para diseñar un SMDP. La dinámica de trabajo establecida con la empresa fue realizada a través de un método de diseño de cinco etapas: definición de los objetivos del SMDP, levantamiento de informaciones, definición de la propuesta de indicadores SPE, validación y evaluación de alternativas de SI para la implementación del SMDP. Durante el proceso de definición de objetivos, es importante asegurar que el concepto de desempeño esperado sea coherente en todos los niveles de la organización, facilitando la identificación de las principales necesidades de medición y de las decisiones que el SMDP deberá soportar. La eficacia de esta etapa depende estrictamente del proceso utilizado y de la participación activa de funcionarios de todos los niveles organizacionales.

Es importante que el levantamiento de informaciones sea realizado a partir de fuentes externas e internas a la organización, para que la visión de los diseñadores de los indicadores cuente con los conocimientos de las mejores prácticas (best-practice) utilizadas en el mercado y del marco operacional y cultural en que el SMDP deberá desarrollarse.

Los indicadores SPE propuestos fueron: Calidad, Entrega, Flexibilidad, y Riesgo Financiero. Las diferencias operacionales y culturales entre los sectores y subsectores del departamento de Compras (bienes, servicios continuos y servicios no continuos) fueron consideradas en la definición de los subindicadores y atributos. De esta manera se permitió que los mismos indicadores sean usados en todos los sectores, manteniendo la homogeneidad y capacidad de comparación del SMDP y evitando la pérdida de los detalles y particularidades de los procesos específicos de compra. El hecho de no permitir que el análisis agregado de los indicadores sea afectado, facilita la toma de decisiones a nivel gerencial y directorio.

El proceso de análisis de las mediciones fue facilitado mediante el uso de símbolos y colores que agilizaban la identificación de niveles y clasificación de los proveedores. El hecho de describir el desempeño de los proveedores a través de todos los indicadores evitó entrar en conflictos de crear subgrupos de proveedores y de determinar qué indicador era más importante para cada subgrupo.

El proceso de validación por medio del uso de prototipos, permite reconocer errores o nuevos requerimientos de implementación en forma oportuna. Revisar y ajustar los indicadores es obligatorio, eso permite la convergencia entre la cultura organizacional y las actividades operacionales recientemente adheridas.

La complejidad de evaluar proveedores crece exponencialmente con el número de variables a controlar. Es recomendado trabajar con un número razonable de indicadores que abarquen los aspectos más significativos del desempeño. Es de esperar que la implementación de un sistema de evaluación genere reacciones adversas en la empresa $\mathrm{y} / \mathrm{o}$ departamentos involucrados. Para quienes ya contaban con alguna forma de evaluación resulta difícil aceptar que tales procesos pueden y deben ser mejorados. Tales obstáculos son mitigados a través de la participación de la alta gerencia. El entendimiento y compromiso de la organización debe provenir de los directores y ser fomentado en los niveles operativos, siendo obligación de los diseñadores del SMDP asegurar que eso se cumpla.

Otro tipo de resistencia puede provenir de los usuarios externos del SMDP, es decir áreas indirectamente involucradas tanto en los procesos de implementación del sistema (departamento de $\mathrm{Tl}$ ) como en los procesos de medición o recolección de datos (almacén, clientes internos). Estas resistencias surgen por la concepción de que existe un aumento en la carga de trabajo, complejidad de las funciones realizadas y en el control de los procesos operativos. Caso los funcionarios no sean incluidos durante todo el proceso de diseño, se corre el riesgo de que la percepción de exceso de control en los niveles operativos generen sabotajes que acaben deteriorando el funcionamiento del SMDP.

El uso de SMDP soporta particularmente el desarrollo y cumplimento de los objetivos del departamento de Compras, los cuales deben ser alineados con los objetivos de la organización. Otros objetivos que conforman la macro estrategia de la 
empresa deben también ser soportados por sistemas de evaluación específicos, los que a su vez, deben ser integrados entre sí, permitiendo que el desempeño de la organización sea visualizado holísticamente. El diseño de tales sistemas de medición puede ser realizado a través de adecuaciones y particularizaciones de la metodología presentada.

\section{Referencias}

BEAMON, B. M. Supply chain design and analysis: Models and methods. International Journal of Production Economics, v. 55, n. 3, p. 281-294, 1998. http://dx.doi. org/10.1016/S0925-5273(98)00079-6

BEAMON, B. M. Measuring supply chain performance. International Journal of Operations \& Production Management, v. 19, n. 3, p. 275-292, 1999. http:// dx.doi.org/10.1108/01443579910249714

BITITCI, U. S.; TURNER, T.; BEGEMANN, C. Dynamics of performance measurement systems. International Journal of Operations and Production Management, v. 20, p. 692-704, 2000. http://dx.doi. org/10.1108/01443570010321676

BOURNE, M. et al. Designing, implementing and updating performance measurement systems. International Journal of Operations and Production Management, v. 20, n. 7, p. 754-771, 2000. http://dx.doi. org/10.1108/01443570010330739

CEBI, F.; BAYRAKTAR, D. An integrated approach for supplier selection. Logistics Information Management, v. $16, \quad$ n. 6, p. 395-400, 2003. http://dx.doi. org/10.1108/09576050310503376

COLLINS, R.; BECHLER, K.; PIRES, S. Outsourcing in the automotive industry: From JIT to Modular Consortia. European Management Journal, v. 15, n. 5, p. 498-508, 1997. http://dx.doi.org/10.1016/S0263-2373(97)00030-3

COYLE, J. J.; BARDI, E. J.; LANGLEY, C. J. R. S. The Management of Business Logistics - A Supply Chain Perspective. Canada: Thomson Learning, 2002.

DO LAGO ATTADIA, L. C.; MARTINS, R. A. Medição de desempenho como base para evolução da melhoria contínua. Revista Produção, v. 13, n. 2, p. 33-41, 2003.

FORTUIN, L. Performance indicators-Why, where and how? European Journal of Operational Research, v. 34, n. 1, p. 1-9, 1988. http://dx.doi.org/10.1016/03772217(88)90449-3

GARENGO, P.; BIAZZO, S.; BITITCl. Performance measurement systems in SMEs: A review for a research agenda. International Journal of Management Reviews, v. 7, n. 1, p. 25-47, 2005. http://dx.doi.org/10.1111/j.14682370.2005.00105.x

GHALAYINI, A. M.; NOBLE, J. S. The changing basis of performance measurement. International Journal of Operations\& Production Management,v. 16, n. 8, p. 63-80, 1996. http://dx.doi.org/10.1108/01443579610125787

GLOBERSON, S. Issues in developing a performance criteria system for an organisation. International Journal of Production Research, v. 23, n. 4, p. 639-646, 1985. http://dx.doi.org/10.1080/00207548508904734
GUNASEKARAN, A.; PATEL, C.; McGAUGHEY, R. E. A framework for supply chain performance measurement. International Journal of Production Economics, v. 87, n. 3, p. 333-347, 2004. http://dx.doi.org/10.1016/j. ijpe.2003.08.003

GUNASEKARAN, A.; PATEL, C.; TIRTIROGLU, E. Performance measures and metrics in a supply chain environment. International Journal of Operations \& Production Management, v. 21, n. 1/2, p. 71-87, 2001. http://dx.doi. org/10.1108/01443570110358468

HARRISON, T. P.; LEE, H. L.; NEALE, J. J. The Practice of Supply Chain Management: Where Theory and Application Converge. 2003. (International Series in Operations Research \& Management Science).

HOEK, R. l. van. Measuring the unmeasurable: measuring and improving performance in the supply chain. Supply Chain Management: An International Journal, v. 3, n. 4, p. 187-192, 1998.

HUDSON, M.; SMART, A.; BOURNE, M. Theory and practice in SME performance measurement systems. International Journal of Operations and Production Management, v. 21, n. 8 , p. 1096-1115, 2000. http://dx.doi. org/10.1108/EUM0000000005587

JUSOH, R.; IBRAHIM, D. N.; ZAINUDDIN, Y. The performance consequence of multiple performance measures usage: Evidence from the Malaysian manufacturers. International Journal of Productivity and Performance Management, v. 57, n. 2, p. 119-136, 2008. http:// dx.doi.org/10.1108/17410400810847393

KAPLAN , R. S.; NORTON, D. P. The Balanced Scorecard - Translating Strategy into Action. Boston: Harvard Business Press, 1996.

KOCABASOGLU, C.; SURESH, N. C. Strategic Sourcing: An Empirical investigation of the concept and its practices in US manufacturing firms. The Journal of Supply Chain Management, v. 42, n. 2, p. 4-15, 2006. http://dx.doi. org/10.1111/j.1745-493X.2006.00008.x

LAMBERT, D. M.; POHLEN, T. L. Supply Chain Metrics. The International Journal of Logistics Management, v. 12, n. 1, p. 1-19, 2001. http://dx.doi. org/10.1108/09574090110806190

LOHMAN, C.; FORTUIN, L.; WOUTERS, M. Designing a performance measurement system: A case study. European Journal of Operational Research, v. 156, n. 2, p. 267-286, 2004. http://dx.doi.org/10.1016/S03772217(02)00918-9

NEELY, A. et al. Performance measurement system design: Should process based approaches be adopted? International Journal of Production Economics, v. 46-47, p. 423-431, 1996. http://dx.doi.org/10.1016/S09255273(96)00080-1

NEELY, A. et al. Designing performance measures: a structured approach. International Journal of Operations \& Production Management, v. 17, n. 11, p. 1131-1152, 1997. http://dx.doi.org/10.1108/01443579710177888

NEELY, A. D. et al. Getting the measure of your Business. Cambridge: Cambridge University Press, 2002.

NEELY, A. D.; GREGORY, M.; PLATTS, K. Performance measurement system design: a literature review and research agenda. International Journal of Operations \& Production Management, v. 15, n. 4, p. 80-116, 1995. http://dx.doi.org/10.1108/01443579510083622 
SHEPHERD, C.; GÜNTER, H. Measuring supply chain performance: current research and future directions. International Journal of Productivity and Performance Management, v. 55, n. 3/4, p. 242-258, 2006. http:// dx.doi.org/10.1108/17410400610653219

SPEKMAN, R. E.; KAMAUFF, J. W.; SALMOND, D. J. At last purchasing is becoming strategic. Long Range Planning, v. 27, n. 2, p. 76 -84, 1994. http://dx.doi. org/10.1016/0024-6301(94)90211-9

TATE, W. L.; van DER VALK, W. Managing the performance of outsourced customer contact centers. Journal of Purchasing and Supply Management, v. 14, n. 3, p. 160-169, 2008. http://dx.doi.org/10.1016/j.pursup.2008.04.002

TRACEY, M.; TAN, C. L. Empirical analysis of supplier selection and involvement, customer satisfaction and firm performance. Supply Chain Management: An International Journal, v. 6, n. 4, p. 174-188, 2001. http://dx.doi.org/10.1108/EUM0000000005709

WAGNER, S. M.; JOHNSON, J. L. Configuring and managing strategic supplier portfolios. Industrial Marketing Management, v. 33, n. 8, p. 717-730, 2004. http:// dx.doi.org/10.1016/j.indmarman.2004.01.005

WISNER, J. D.; FAWCETT, S. E. Link firm strategy to operating decisions through performance measurement. Production and Inventory Management Journal, n. Terceiro Trimestre, p. 5-11, 1991.

WISNER, J. D.; STANLEY, L. L. Process Management: Creating Value Along the Supply Chain; Texts \& Cases. Thomson South-Western, 2007.

\begin{abstract}
The evaluation of suppliers' performance is important for organizations because of the imperative role they play in the supply chains and the importance that purchasing functions have due to the outsourcing of activities which are not part of the company's know-how. There is a need to work on the identification of practical problems concerning the design and implementation of supplier performance measurement systems (SPMS). This paper presents the most important factors in designing and implementing such systems through an action research in a multinational media enterprise. In addition to the details of the method and results of its application, the paper presents an analysis of processes and attributes that define the design of the proposed system. The authors aim to contribute to the academic literature in providing practical issues regarding SPMS.
\end{abstract}

Design of supplier performance measurement systems: experiences from an action research

\title{
Keywords
}

Performance measurement systems. Supply chain. Suppliers. Supplier performance evaluation. 